\title{
Intraseptal Procaine Abolishes Hypothalamic Stimulation-Induced Wheel-Running and Hippocampal Theta Field Activity in Rats
}

\author{
Scott D. Oddie, Wendy Stefanek, lan J. Kirk, and Brian H. Bland \\ Department of Psychology, Behavioral Neuroscience Research Group, University of Calgary, Calgary, \\ Alberta, Canada T2N 1N4
}

Rats were implanted chronically with hippocampal recording electrodes, a microinfusion guide cannula aimed at the medial septal nucleus, and an electrode for electrical stimulation of the posterior hypothalamic nucleus (PH). $\mathrm{PH}$ stimulation elicited running in rats placed in a wheel and simultaneously occurring hippocampal theta field activity (HPC- $\theta)$. In the preprocaine (PRE) testing condition, a positive linear relationship was demonstrated among the intensity of electrical stimulation of the $\mathrm{PH}$, wheel-running speed, and the peak frequency of HPC- $\theta$. HPC- $\theta$ amplitude reached an asymptote at the lowest levels of electrical stimulation of the $\mathrm{PH}$. Procaine hydrochloride $(1.5 \mu \mathrm{l}$, $20 \%$ solution), a local anesthetic, was then infused into the medial septal nucleus (MS). Five minutes after the infusion, $\mathrm{PH}$ stimulation no longer induced wheel-running behavior or HPC- $\theta$, and the remaining irregular field activity was significantly reduced in amplitude. Fifteen minutes after the procaine infusion, PH stimulation still did not elicit HPC- $\theta$ or running behavior in the majority of animals but did evoke largeamplitude sharp-waves. Thirty minutes after the procaine infusion, $\mathrm{PH}$ stimulation again elicited HPC- $\theta$ and running behavior, but HPC- $\theta$ peak frequency and running speeds were both significantly reduced compared with PRE values. Forty-five minutes after the infusion, HPC- $\theta$ amplitude had recovered to PRE values, but HPC- $\theta$ frequency and running speeds elicited by $\mathrm{PH}$ stimulation were still significantly reduced. By $60 \mathrm{~min}$ after procaine administration, the amplitude and frequency of HPC- $\theta$ and the running speeds elicited by $\mathrm{PH}$ stimulation recovered to PRE values. Multiple regression analysis revealed that the recovery pattern of running behavior reflected the frequency rather than the amplitude of HPC- $\theta$. Neither saline control infusions into the MS nor procaine infusions into the lateral septum and paraventricular thalamic nucleus affected HPC- $\theta$ or running behavior. These findings are consistent with the notion that both the locomotor activity and "movementrelated" HPC- $\theta$ frequency induced by electrically stimulating the $\mathrm{PH}$ were attributable to ascending activation of a hypothalamo-septal pathway and not to activation of descending brainstem or peripheral motor systems.

Key words: posterior hypothalamus; medial septum; procaine inactivation; hippocampal theta field activity; wheel-running behavior; ascending brainstem synchronizing pathway
Hippocampal theta field activity (HPC- $\theta$ ) is a rhythmic field potential that oscillates at a frequency between 3 and $12 \mathrm{~Hz}$ and has been suggested to be involved in a variety of theories of hippocampal function including attention and motivation (Green and Arduini, 1954; Grastyan et al., 1959, 1966; Bennett et al., 1973), mechanisms of learning and memory (Elazar and Adey, 1967; O'Keefe and Nadel, 1978; Rose and Dunwiddie, 1986; Grcenstein et al., 1988; Pavlides et al., 1988; Buzsaki, 1989; Alonso et al., 1990; Eichenbaum et al., 1992; Huerta and Lisman, 1993; Markowska et al., 1995; Staubli and Xu, 1995), and integration of motor programming (Vanderwolf, 1969; Black, 1975; Thompson et al., 1980; Morris and Hagan, 1983; Lopes Da Silva et al., 1985; Bland, 1986). In behaving animals, HPC- $\theta$ is present during "exploratory" behaviors, paradoxical and slow-wave sleep (Gatztelu et al., 1994), and accompanies "voluntary" motor behaviors such as walking, running, rearing, and head movements (Vanderwolf, 1969). During nonalert immobility and when animals are engaged in "automatic" motor activities such as chewing, licking, grooming, or shivering, large-amplitude irregular activity

Received Sept. 14, 1995; revised Nov. 9, 1995; accepted Nov. 22, 1995.

This work was supported by Natural Sciences and Engineering Research Council Grant A9935 to B.H.B., a University of Calgary Postdoctoral Fellowship to I.J.K., and an Alberta Heritage Foundation for Medical Research Postgraduate Scholarship to S.D.O.

Correspondence should be addressed to Dr. Brian H. Bland at the above address. Copyright $\subset 1996$ Society for Neuroscience $0270-6474 / 96 / 161948-09 \$ 05.00 / 0$ is recorded in the hippocampal formation (Vanderwolf, 1969; Whishaw and Vanderwolf, 1973).

The medial septal nucleus (MS) is the primary extrinsic regulator of HPC- $\theta$ (Stewart and Fox, 1990). In unanesthetized animals, lesions of the MS abolish HPC- $\theta$ (Green and Arduini, 1954; Petsche and Stumpf, 1960; Petsche et al., 1962; Donovick, 1968; Gray, 1971; Myhrer, 1975; Kolb and Whishaw, 1977; Winson, 1978; Andersen et al., 1979; Monmaur et al., 1979; Rawlins et al., 1979; Sainsbury and Bland, 1981). Transection of the fimbria/ fornix, which connects the MS to the hippocampal formation, also abolishes HPC- $\theta$ (Green and Arduini, 1954; Ranck, 1973; Buzsaki et al., 1983). Electrical stimulation of the MS, when delivered in theta-patterned bursts, elicits theta-like activity in the hippocampal formation; however, the behavior of the animals during MS stimulation is uncorrelated with the induced HPC- $\theta$ (Kramis and Routtenberg, 1977). Conversely, septal inactivation, achieved via the microinfusion of a local anesthetic directly into the MS, temporarily abolishes HPC- $\theta$ and reduces the occurrence of locomotor activity (Brücke et al., 1959; Mizumori et al., 1989; Lawson and Bland, 1993).

Electrical stimulation at various levels of the brainstem reticular formation and caudal diencephalon elicits HPC- $\theta$. Increasing the intensity of stimulation in these areas elicits increasing frequencies of HPC- $\theta$ (Torii, 1961; Yokota and Fujimori, 1964; Grastyan et al., 1966; Bland and Vanderwolf, 1972a; Klemm, 
1972a,b; Macadar et al., 1974; Paiva et al., 1976; Robinson and Vanderwolf, 1978; Vertes, 1980, 1981). The synchronizing effect these regions have on hippocampal field activity may be mediated via the MS and originate in the rostral pons region (PO) (Macadar et al., 1974; Vertes, 1980, 1981). Fibers from the PO reach the MS via nuclei in the caudal diencephalon, namely the supramamillary nucleus and the posterior hypothalamic nucleus (Vertes, 1982, 1984, 1988); both nuclei project to the MS (Vertes, 1992; Vertes et al,, 1995). The discharge patterns of cells in these nuclei show distinct relationships to hippocampal field activity (Kirk and McNaughton, 1991; Nunez et al., 1991; Kocsis and Vertes, 1994; Bland et al., 1995), and the infusion of a local anesthetic in this area blocks the induction of HPC- $\theta$ with PO stimulation (Kirk and McNaughton, 1993; Oddie et al., 1994).

Elcctrical stimulation of the posterior hypothalamic nucleus $(\mathrm{PH})$ not only elicits HPC- $\theta$, but also induces locomotor activity; however, the motor behavior is dependent on the environmental situation (Gerben, 1968, 1969; Bland and Vanderwolf, 1972a; Whishaw et al., 1972). The same intensity of PH stimulation can induce a rat to run in a wheel, jump out of a box, dig in bedding, or explore a novel environment. Increasing the intensity of $\mathrm{PH}$ stimulation not only increases the frequency of HPC- $\theta$, but also increases the intensity with which motor programs are initiated and maintained in each situation (e.g., increasing running speeds are effected by increasing intensities of stimulation). In freely moving rats, $\mathrm{PH}$ lesions decrease the frequency of HPC- $\theta$ and the occurrence of voluntary motor behavior while leaving "automatic" movements intact (Robinson and Whishaw, 1974).

Taken collectively, these studies indicate that the $\mathrm{PH}$ may exert its influence on both HPC $-\theta$ and concomitant locomotor activity via a hypothalamo-septal pathway. Alternatively, $\mathrm{PH}$ stimulation may induce movement by activating descending motor systems. Locomotor activity elicited in this manner would then cause "movement-related" HPC- $\theta$. In this experiment, rats were implanted with hippocampal recording electrodes and induced to run in a wheel with electrical stimulation of the PH. Procaine, a local anesthetic, was then infused into the medial septum, and its effect on the previously elicited behavior and field activity was examined. In the event that MS procaine abolished HPC- $\theta$, and had no influence on the PH elicited motor behavior, the alternative hypothesis would be confirmed. Conversely, if MS procaine abolished both HPC- $\theta$ and the elicited wheel-running behavior, then the aforementioned hypothesis would be confirmed; that is, PH-elicited behavior and the accompanying HPC- $\theta$ are elicited by activating an ascending hypothalamo-septal pathway that synchronizes the hippocampal field activity and plays a role in sensorimotor integration (see also Bland and Colom, 1993).

\section{MATERIALS AND METHODS}

Animals. Male Long-Evans rats $(300-550 \mathrm{gm})$ were used. The animals were supplied by the University of Calgary Animal Vivarium, housed individually, and maintained on a $12 \mathrm{hr}$ light/dark cycle. Fond and water were provided ad libitum.

Chronic implant procedure. Animals were implanted chronically with bilateral hippocampal recording electrodes, a guide tube aimed at the medial septum, and a bipolar electrode for electrical stimulation of the PH. Each animal was given atropine methyl nitrate $(10 \mathrm{mg} / \mathrm{kg})$ and then anesthetized with sodium pentobarbital $(65 \mathrm{mg} / \mathrm{kg})$. The animal was placed in a stereotaxic instrument, and bregma and lambda were leveled to horizontal. An uninsulated tungsten wire implanted into the cortex anterior to bregma served as an indifferent electrode. Tungsten microelectrodes $(0.2-0.8 \mathrm{M} \Omega)$ were placed bilaterally into the stratum moleculare of the fascia dentata $(3.3 \mathrm{~mm}$ posterior to bregma, $2.4 \mathrm{~mm}$ lateral to the midline, and $2.7 \mathrm{~mm}$ ventral to the dural surface). A guide tube, constructed of $14.6-\mathrm{mm}$-long 23-gauge stainless steel tubing, was im- planted $2.7 \mathrm{~mm}$ ventral to the dural surface. The guide tube positioned a microinfusion cannula, constructed of $17.6-\mathrm{mm}$-long 30 -gauge stainless steel tubing, in the medial septum. The tip of the microinfusion cannula extended $3.0 \mathrm{~mm}$ beyond the guide tube to $5.7 \mathrm{~mm}$ ventral to the dural surface. A bipolar stimulation electrode (MS 303/1, Plastics One, Roanoke, VA), $\sim 250 \mu \mathrm{m}$ in diameter, was implanted into the PH $3.5 \mathrm{~mm}$ posterior to bregma, $0.0-0.2 \mathrm{~mm}$ lateral the midline, and $7.6 \mathrm{~mm}$ ventral to the dural surface. A subminiature connector soldered to a panhead screw and placed in the skull adjacent to lambda served as a ground electrode. The electrodes and guide tube were held in place using dental acrylic fixed to panhead screws placed in the skull. The recording, ground, and indifferent electrodes were then connected to a female 4-pin Amphenol plug that was fixed to the skull using dental acrylic. The animals were given a minimum 1 week recovery period before testing began. Thirteen animals that had stable chronic recording and stimulation arrangements were involved in the behavioral testing described below.

$H P C$ recording, $P H$ electrical stimulation, and MS infusion procedure. Signals from the brain were amplified by an operational amplificr set at $10 \times$ gain (LF 444 CM, Linear Technology, Milpitas, CA) located in a 4-pin male Amphenol plug that was connected to the animal. Signals passed through a cable into a commutator that then fed to band-pass $(1-35 \mathrm{~Hz})$ Grass P511 AC preamplifiers. The signals were displayed on a storage oscilloscope and stored on an FM tape recorder for subsequent analysis. The experimenter's vocal description of ongoing behavior during testing was also recorded on the FM tape. In the preprocaine (PRE) condition, electrical stimulation of the $\mathrm{PH}$ was delivered in a block containing two sets of three stimulus intensities $(0.1 \mathrm{msec}$ duration, 100 $\mathrm{Hz}$ square wave). Stimulus intensities varied for each animal and were chosen using the following criteria: (1) the lowest intensity that elicited wheel-running behavior, and (2) two higher intensities at $0.1-0.2 \mathrm{~mA}$ increments from the lowest intensity. Stimulus intensities ranged from 0.1 to $1.0 \mathrm{~mA}$ and were delivered to thie animal via the commutator using a Grass S88 stimulator equipped with a stimulus isolation unit. Any given stimulus intensity within a block lasted $\sim 10 \mathrm{sec}$ and was followed by a 10-30 sec delay. Thus, a stimulation block contained six $10 \mathrm{sec}$ stimulus bouts delivered to the $\mathrm{PH}$, two at each of the three intensities. In the drug testing condition, a solution of procaine hydrochloride in physiological saline $(20 \%)$ was infused into the MS. The microinfusion cannula was connected to a $10 \mu \mathrm{l}$ Hamilton Gas-Tight syringe (Reno, NV) via a length of PE 50 intramedic tubing that contained the procaine solution. The syringe was mounted on a Harvard Apparatus (Boston, MA) infusion pump that delivered $0.5 \mu \mathrm{l} / \mathrm{min}$ for $3 \mathrm{~min}$. The microinfusion cannula was not removed until $3 \mathrm{~min}$ after the termination of the MS infusion. Control infusions used only physiological saline. After drug or control infusions, a stimulation block was delivered to the $\mathrm{PH}$ nucleus for each of the postprocaine experimental conditions as in the PRE testing condition.

Apparatus and behavioral testing procedure. The experiments were conducted in a running-wheel ( $43 \mathrm{~cm}$ diameter) placed in a Faraday cage $\left(122 \times 65 \times 65 \mathrm{~cm}^{3}\right)$. The running wheel was equipped with a speedometer (Cateye Mity Cyclocomputer, Osaka, Japan, model CC-MT100), which converted wheel revolutions to $\mathrm{km} / \mathrm{hr}$ hased on the wheel diameter The speedometer recorded the maximum speed achieved during $\mathrm{PH}$ stimulation. A testing session began by connecting the $\mathrm{PH}$ stimulation and hippocampal recording leads to the animal and then placing the animal in the running-wheel. The lowest level of PH stimulation that induced wheel-running was determined, and then two higher levels were chosen. The stimulus levels were input into a PC equipped with a BASIC program that generated six blocks of randomly ordered stimulus intensities: one block for each of the PRE, 5 min postprocaine (P5), 15 min postprocaine (P15), 30 min postprocaine (P30), $45 \mathrm{~min}$ postprocaine $(\mathrm{P} 45)$, and $60 \mathrm{~min}$ postprocaine (P60) experimental conditions. In the PRE condition, a stimulus block was delivered to the animal. For each intensity in the block, the maximum running speed elicited by the $\mathrm{PH}$ stimulation was recorded on the FM tape along with the simultaneously occurring hippocampal field activity. The speedometer was then zeroed, and the subsequent stimulus intensities were administered. After the PRE condition, the procaine microinfusion cannula was inserted into the guide tube. Procaine hydrochloride or saline was infused into the MS, and 5 min after the termination of the infusion the P5 stimulation block was delivered to the PH and the running speeds and simultaneously occurring hippocampal field activity were recorded. The same procedure followed for the P15-P60 experimental conditions. Behavior and hippocampal field activity were also recorded using a video recorder and a split-screen technique. One camera recorded the oscilloscope display of the hippocampal field activity while a second camera recorded ongoing behavior. 
Figure 1. Procaine microinfusions in the MS. Diagrammatic reconstructions illustrating the location of the medial septal microinfusion cannula tips. The infusions were localized to three coronal planes: $0.45 \mathrm{~mm}$ anterior to bregma $(A), 0.10 \mathrm{~mm}$ anterior to bregma $(B)$, and $0.0 \mathrm{~mm}$ anterior to bregma $(C)$. All stimulation electrodes were in the $\mathrm{PH}$ or within close proximity; an example is provided in $D$. The diagram in the top left panel shows the location of the coronal sections in a midsagittal view. $a c$, Anterior commissure; $c c$, corpus callosum; $C M$, central medial nucleus of the thalamus; $D M H$, dorsal medial hypothalamic nucleus; $H D B$, horizontal limb of the diagonal band; $L S$, lateral septal nucleus; $M S$, medial septal nucleus; $o c$, optic chiasm; $V D B$, vertical limb of the diagonal band.
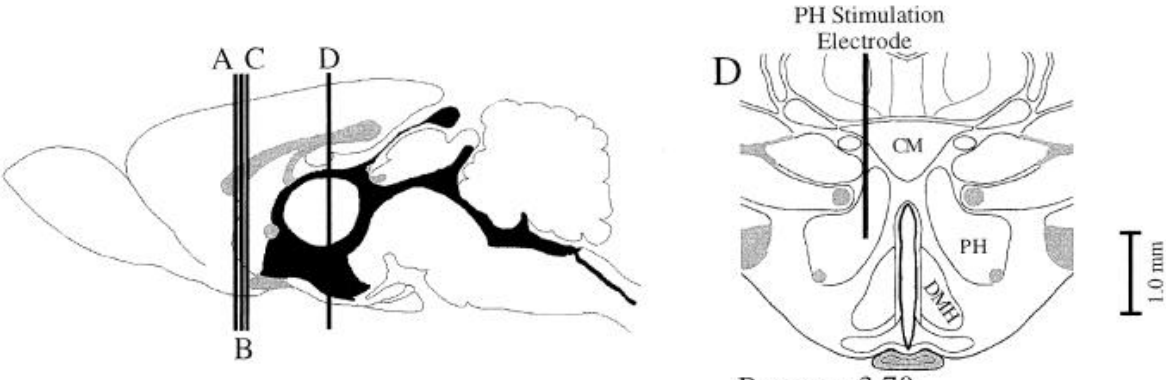

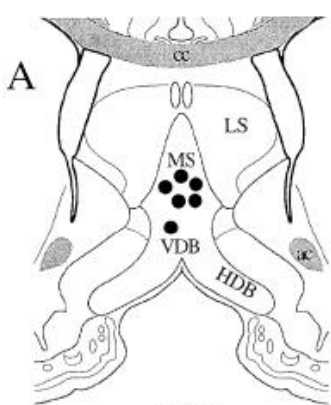

Bregma +0.45

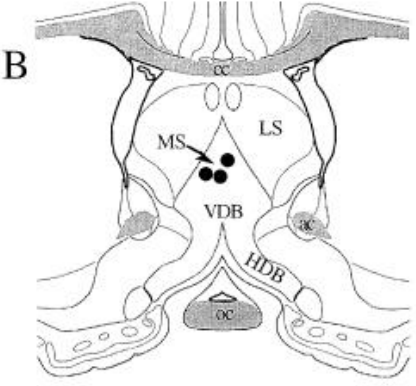

Bregma +0.10
Bregma -3.70

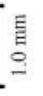

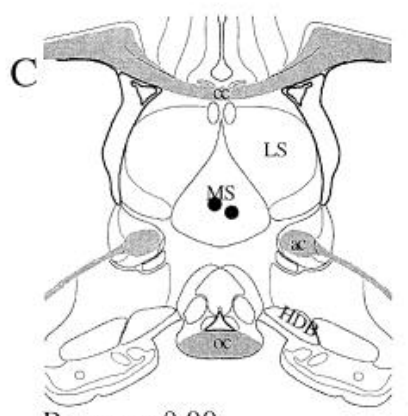

Bregma -0.00
The two video inputs were recorded simultaneously and viewed by the experimenter during testing. Thirteen animals were tested with procaine microinfusions. Three of these animals also served as controls and, in addition to procaine, received physiological saline infusions. After perfusion and fixation of the brain, frozen sections $(40 \mu \mathrm{m})$ were taken serially and mounted on glass slides and stained with cresyl violet for subsequent verification of hippocampal field electrode, microinfusion cannula, and $\mathrm{PH}$ stimulation electrode tip placements.

Data analysis. Analysis of the data segments was accomplished off-line using a PC and a software acquisition and analysis package (DataWave Technologies, Longmount, CO). A data segment was collected during each level of PH stimulation (6-8 sec of data were selected from within the $10 \mathrm{sec}$ stimulus train). Hippocampal field activity was sampled at a frequency of $133 \mathrm{~Hz}$. Each data segment was subjected to a real-time fast Fourier (FFT) analysis, and the peak frequency and amplitude values were recorded. Theta was defined as a sinusoidal-like waveform with a peak frequency of $3-12 \mathrm{~Hz}$ and a small bandwidth. If there was no prominent peak in the spectrum, the largest power value in the $3-12 \mathrm{~Hz}$ range was recorded. The maximum running speed achieved during the 10 sec of PH stimulation was also recorded. The peak frequency, amplitude, and running speed values elicited by $\mathrm{PH}$ stimulation at the same intensity within a block were averaged. A one-way repeated-measures ANOVA was used to analyze each of the peak frequency, amplitude, and running speed measures. Multiple regression and Dunnett's $t$ test for pairwise comparisons were used for post hoc analyses. Histological illustrations were generated using the computer graphics files that accompany Brain Maps (Swanson, 1992).

\section{RESULTS}

\section{Histology}

In all animals tested, hippocampal recording electrodes were localized to the molecular layer of the dentate gyrus. Figure 1 illustrates the location of the microinfusion cannula in 11 of 13 animals tested with procaine hydrochloride $(A-C)$. The microinfusion cannula tips were localized to the MS in three coronal planes: $0.45 \mathrm{~mm}$ anterior to bregma $(A), 0.10 \mathrm{~mm}$ anterior to bregma $(B)$, and $0.00 \mathrm{~mm}$ anterior to bregma $(C)$. Figure 1 (top left panel) shows the location of the coronal sections in a mid-sagittal view. Figure $1 D$ illustrates the placement of a $\mathrm{PH}$ stimulation electrode. All PH stimulation electrode tips were located in the $\mathrm{PH}$ (3.4-3.7 $\mathrm{mm}$ posterior to bregma and $0.0-0.9 \mathrm{~mm}$ lateral to midline). In 2 of 13 animals, the microinfusion cannula was not localized to the MS, and the data from these animals served as histological controls (see below).

\section{$\mathrm{PH}$ electrical stimulation and procaine microinfusion in the medial septum}

Figure 2 provides a representative example from one animal illustrating the effect of procaine microinfusion into the MS on HPC- $\theta$ and wheel-running elicited by $0.15 \mathrm{~mA}$ PH stimulation. Data segment analogs of the hippocampal field activity at the onset of PH stimulation (solid bar) are shown. Figure 2 (middle graph) also provides the corresponding spectra from FFT analysis for each data segment in the PRE-P60 experimental conditions (spectra plot linear values). Before the microinfusion of procaine in the MS (PRE), electrical stimulation in the PH elicited HPC- $\theta$ with an amplitude of $55.8 \mathrm{~dB}$ and a peak frequency of $10.0 \mathrm{~Hz}$ and induced a maximum running speed of $17.0 \mathrm{~km} / \mathrm{hr}$. At P5, the same intensity of PH stimulation no longer elicited HPC- $\theta$ (absence of peak in the P5 spectrum) or induced wheel-running behavior (running speed $=0.0$ ). At P15, PH stimulation still did not elicit HPC- $\theta$ or wheel-running behavior (absence of peak in the P15 spectrum) but, rather, evoked large amplitude sharp waves in the hippocampal field. At P30, PH stimulation again elicited HPC- $\theta$, but at a reduced amplitude and frequency, and induced wheelrunning at speeds that were also reduced compared with PRE values. In the P30 condition, $0.15 \mathrm{~mA}$ PH stimulation elicited HPC- $\theta$ with an amplitude of $35.1 \mathrm{~dB}$, a peak frequency of $7.8 \mathrm{~Hz}$, and induced a maximum running speed of $13 \mathrm{~km} / \mathrm{hr}$. Although the amplitude of HPC- $\theta$ had nearly recovered to PRE values at MS P45 (53.5 dB at P45), the peak frequency of HPC- $\theta$ and running speed elicited by $\mathrm{PH}$ stimulation were still reduced $(8.3 \mathrm{~Hz}$ and 14 $\mathrm{km} / \mathrm{hr}$, respectively). By MS P60, the amplitude of HPC- $\theta$ had recovered to PRE values $(55 \mathrm{~dB})$, and in this animal the peak frequency HPC- $\theta$ and running speed had nearly recovered to PRE values $(9.1 \mathrm{~Hz}$ and $14 \mathrm{~km} / \mathrm{hr}$, respectively).

Behaviorally, the microinfusion of procaine into the MS resulted in animals that appeared catatonic, but when handled they moved voluntarily without overt postural abnormalities. Figure 3 


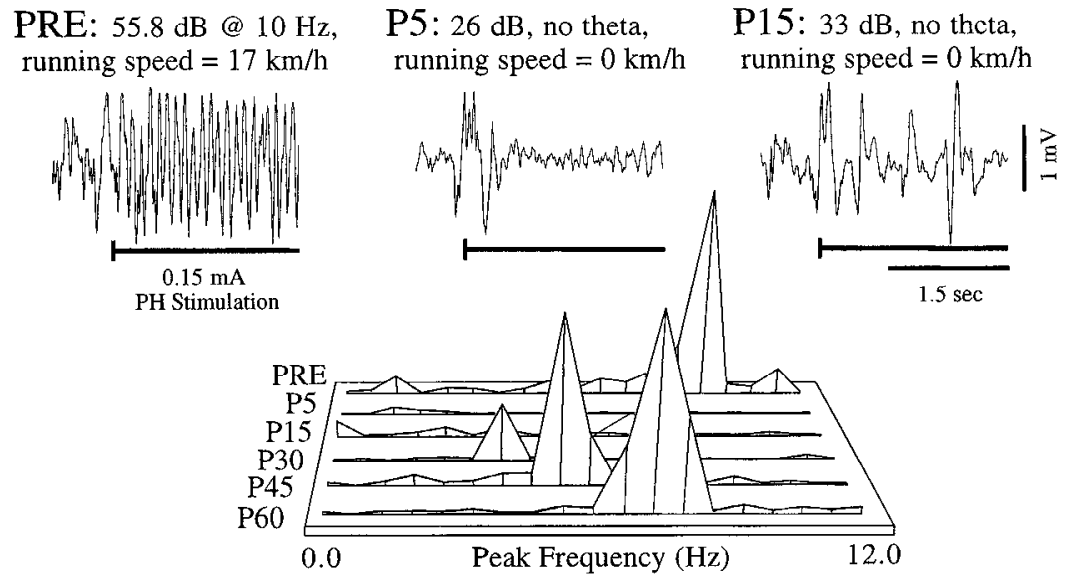

P30: $35.1 \mathrm{~dB} @ 7.8 \mathrm{~Hz}$, running speed $=13 \mathrm{~km} / \mathrm{h}$

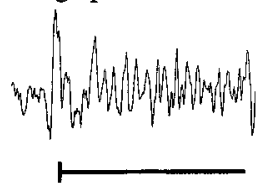

P45: $53.5 \mathrm{~dB} @ 8.3 \mathrm{~Hz}$, running speed $=14 \mathrm{~km} / \mathrm{h}$

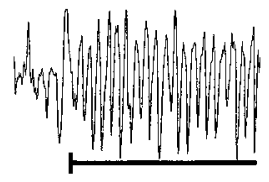

P60: $55 \mathrm{~dB} @ 9.1 \mathrm{~Hz}$ running specd $=14 \mathrm{~km} / \mathrm{h}$

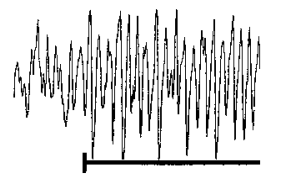

Figure 2. The effect of medial septal procaine on hippocampal field activity and running speeds induced by electrical stimulation of the posterior hypothalamus $(0.15 \mathrm{~mA})$ : representative recordings and graphic summary (stimulation onset indicated by solid bars). The middle graph provides the spectra caused by FFT analyses of the data segments (only partial data segments are shown) for each of the experimental conditions (graph plots linear values). Before the infusion of procaine into the medial septum $(P R E), P H$ Stimulation induced a running speed of $17 \mathrm{~km} / \mathrm{hr}$ and simultaneously occurring HPC- $\theta$ with a peak frequency of $10 \mathrm{~Hz}$ and an amplitude of $55.8 \mathrm{~dB}$ (see the corresponding data analog and FFT spectrum for the PRE condition). Five minutes after the infusion of procaine into the medial septum $(P 5)$, the same intensity of PH stimulation no longer induced running or elicited hippocampal theta (no peak in spectrum at $\mathrm{P5}$ ), and the remaining field activity was reduced to $26 \mathrm{~dB}$. The frequency and amplitude of HPC- $\theta$ gradually recovered to PRE values, and by $60 \mathrm{~min}$ after procaine administration $(P 60), 0.15 \mathrm{~mA} \mathrm{PH}$ stimulation induced running at $14 \mathrm{~km} / \mathrm{hr}$ and simultaneously occurring theta with a peak frequency of $9.1 \mathrm{~Hz}$ and an amplitude of $55 \mathrm{~dB}$. shows the group data obtained from 11 animals that received procaine microinfusion in the MS at each of the three PH stimulation levels used. The $\mathrm{PH}$ stimulation values shown in the legend of Figure 3 are the averages for each of the low (mean 0.28 $\mathrm{mA}$ ), medium (mean $0.41 \mathrm{~mA}$ ), and high (mean $0.54 \mathrm{~mA}$ ) intensity levels chosen for each animal.

\section{The effect of MS procaine on the amplitude of hippocampal theta elicited by PH electrical stimulation}

Figure 3 (top graph) shows the effect of MS procaine on the amplitude of hippocampal field activity. After MS inactivation, PH stimulation no longer elicited HPC- $\theta$. A one-way repeatedmeasures ANOVA revealed that MS procaine had significantly reduced the amplitude of the remaining irregular hippocampal field activity during $\mathrm{PH}$ stimulation $\left(F_{(5,10)}=54.6, p<0.0001\right)$. The main effect of $\mathrm{PH}$ stimulation intensity on the amplitude of HPC- $\theta$ was not significant. The average amplitude of $\mathrm{HPC}-\theta$ (averaged across all levels of $\mathrm{PH}$ stimulation) before the microinfusion of procaine in the MS was $47.7 \pm 1.4 \mathrm{~dB}( \pm \mathrm{SEM})$. After the microinfusion of procaine into the MS, HPC- $\theta$ was abolished and the average amplitude of the remaining irregular hippocampal field activity (when measured as the largest amplitude within the theta bandwidth) was $30.1 \pm 1.8 \mathrm{~dB}$. Thus, 5 min after procaine infusion into the MS, the amplitude of the hippocampal field activity during $\mathrm{PH}$ stimulation was reduced by $\sim 1.0 \mathrm{mV}$. Figure 3 (top graph) also shows that in the earlier period of MS recovery, the amplitude of $\mathrm{HPC}-\theta$ was rapidly recovering to $\mathrm{PRE}$ values $(\mathrm{P} 15,35.9 \pm 1.8 \mathrm{~dB} ; \mathrm{P} 30,38.7 \pm 1.7 \mathrm{~dB} ; \mathrm{P} 45,42.0 \pm 1.8$; $\mathrm{P} 60,44.1 \pm 1.6 \mathrm{~dB})$. There was no significant difference in the amplitude of HPC- $\theta$ elicited by the highest level of PH stimulation at the PRE and P60 conditions, indicating that the MS had recovered from the procaine microinfusion ( $48.2 \pm 2.5 \mathrm{~dB}$ PRE vs $45.2 \pm 3.0 \mathrm{~dB}$ P60; $\left.t_{(10)}=2.1, p=0.06\right)$.

\section{The effect of MS procaine on the peak frequency of hippocampal theta elicited by PH electrical stimulation}

Figure 3 (middle graph) shows the group data for the effect of procaine microinfusion into the $\mathrm{MS}$ on the peak frequency of HPC- $\theta$. In the PRE condition, increasing the intensity of $\mathrm{PH}$ stimulation significantly increased the peak frequency of HPC- $\theta$ $\left(t_{(32)}=-64.6, p<0.0001\right)$. PH stimulation at the low intensity $(0.28 \mathrm{~mA})$ elicited hippocampal theta with an average peak frequency of $8.4 \pm 0.1 \mathrm{~Hz}, 8.9 \pm 0.2 \mathrm{~Hz}$ at the medium intensity $(0.41$ $\mathrm{mA}$ ), and $9.4 \pm 0.3 \mathrm{~Hz}$ at the high intensity (0.54 mA). At P5, HPC- $\theta$ was completely abolished in 11 of 11 animals at the lowest level of PH stimulation $(0.28 \mathrm{~mA})$, abolished in 10 of 11 animals at the medium $\mathrm{PH}$ intensity $(0.41 \mathrm{~mA})$, and abolished in 9 of 11 animals at the highest intensity of $\mathrm{PH}$ stimulation $(0.54 \mathrm{~mA})$. Thus, the values plotted in Figure 3 (middle graph) for the P5 condition (right of dashed line) are peak frequency values in only 1 animal at the medium $(0.41 \mathrm{~mA})$ intensity of $\mathrm{PH}$ stimulation and 2 animals at the highest $(0.54 \mathrm{~mA})$ intensity of $\mathrm{PH}$ stimulation. The peak frequency of HPC- $\theta$ (in the 3 animals in which theta was recorded in the $\mathrm{P} 5$ condition during $\mathrm{PH}$ stimulation) was reduced compared with PRE values. At P15, PH stimulation elicited HPC $-\theta$ in only 2 of 11 animals at the low $(0.28 \mathrm{~mA}) \mathrm{PH}$ intensity, in 9 of 11 animals at the medium $(0.41 \mathrm{~mA}) \mathrm{PH}$ intensity, and in 9 of 11 animals at the high $(0.54 \mathrm{~mA})$ intensity. The frequency of HPC- $\theta$ in these animals was reduced compared with PRE values. Only large-amplitude hippocampal sharp-waves were recorded at this time. At P30, PH stimulation elicited HPC- $\theta$ in 6 of 11 animals at the $0.28 \mathrm{~mA}$ intensity, in 11 of 11 animals at the 0.41 $\mathrm{mA}$ intensity, and in 11 of 11 animals at the $0.54 \mathrm{~mA}$ intensity. The frequency of HPC- $\theta$ remained at values that were reduced compared with PRE values. At P45, PH stimulation elicited $\mathrm{HPC}-\theta$ in 8 of 11 animals at the $0.28 \mathrm{~mA}$ intensity, in 11 of 11 animals at the $0.41 \mathrm{~mA}$ intensity, and in 11 of 11 animals at the $0.54 \mathrm{~mA}$ intensity; the frequency of HPC- $\theta$ remained reduced compared with PRE values. At P60, PH stimulation elicited HPC- $\theta$ in 9 of 11 animals at the $0.28 \mathrm{~mA}$ intensity, in 11 of 11 animals at the 0.41 $\mathrm{mA}$ intensity, and in 11 of 11 animals at the $0.54 \mathrm{~mA}$ intensity. The frequency of HPC- $\theta$ in these animals was still slightly reduced compared with PRE values. In 2 animals in the $\mathrm{P} 60$ condition, no HPC- $\theta$ was recorded at the lowest PH stimulation level.

Given the nature of the effect of MS procaine on the peak frequency of HPC- $\theta$, the data could not be analyzed using repeated-measures ANOVA because of the large number of 

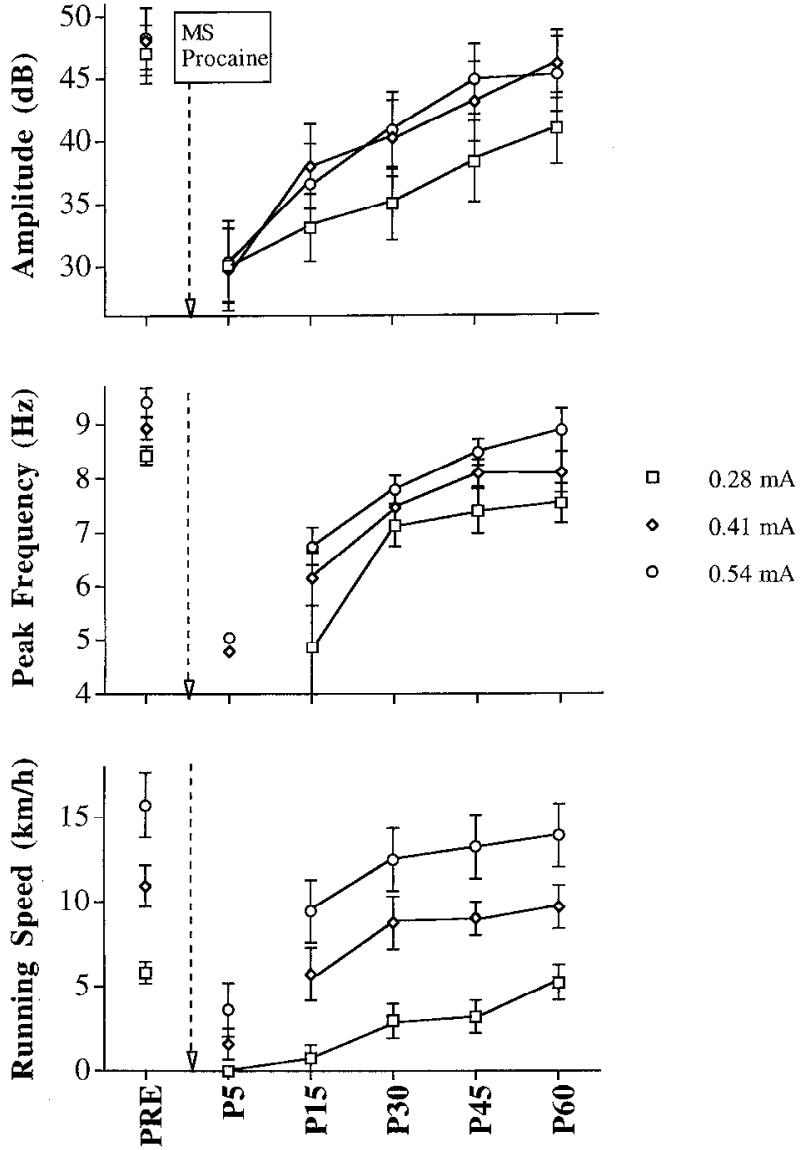

Figure 3. The effect of medial septal procaine infusion on theta and running speed. Group data on the amplitude (top) and peak frequency (middle) of HPC- $\theta$ and running speed (bottom) induced by electrical stimulation delivered to the $\mathrm{PH}$. The $\mathrm{PH}$ stimulation values shown in this legend are the averages for each of the low $(\bar{x}=0.28 \mathrm{~mA} ; \sqcap)$, medium $(\bar{x}$ $=0.41 \mathrm{~mA} ; \diamond)$, and high $(\bar{x}=0.54 \mathrm{~mA} ; \bigcirc)$ intensity levels chosen for each animal. Before the infusion of procaine into the medial septum, increasing the intensity of posterior hypothalamic stimulation had no significant effect on the amplitude of HPC- $\theta$ (top, left of dashed line, MS Procaine), significantly increased the peak frequency of HPC- $\theta$ (middle, left of dashed line), and significantly increased the speed of running (bottom, left of dashed line). After MS procaine, the amplitude of hippocampal field activity was significantly reduced (P5), HPC- $\theta$ could no longer be elicited by $\mathrm{PH}$ stimulation in most animals (see Results), and running could no longer be elicited by PH stimulation. As the medial septum recovered from the procaine infusion the amplitude and frequency of hippocampal field activity gradually recovered to PRE values, as did the speed of running induced by each PH stimulus level. There was no longer any significant difference between the amplitudes and frequencies of HPC- $\theta$ or the running speeds induced at the PRE and P60 experimental conditions, indicating that the MS had recovered from the procaine inactivation.

missing cells in the P5 and P15 conditions. That is, the failure to observe a peak in the FFT analysis of a data segment during $\mathrm{PH}$ stimulation (indicating that theta had been abolished) caused a missing data cell. Thus, to examine the repeated-measure effect of MS procaine on the frequency of HPC- $\theta$, the following data analysis was performed. If HPC- $\theta$ was elicited by the highest intensity of $\mathrm{PH}$ stimulation in any of the postprocaine experimental conditions, the peak frequency values were plotted for each of the remaining experimental conditions and the slope of those values (which represent recovery of the MS from the procaine microinfusion) for each animal was determined. A one-sample $t$ test revealed that the values were significantly different from zero, indicating that the peak frequency of HPC- $\theta$ changed significantly as the MS recovered from procaine inactivation $\left(t_{(10)}=4.9, p<\right.$ 0.0006 ; mean slope $=0.7$ ). The peak frequency of HPC- $\theta$ was not significantly different between the PRE and P60 conditions at the highest level of $\mathrm{PH}$ stimulation $(9.4 \pm 0.3 \mathrm{~Hz} \mathrm{PRE}$ vs $8.9 \pm 0.4 \mathrm{~Hz}$ $\left.\mathrm{P} 60 ; t_{(10)}-0.97, p=0.35\right)$, indicating that the MS had recovered from the procaine microinfusion.

\section{The effect of MS procaine on the speed of wheel- running induced by PH electrical stimulation}

Figure 3 (bottom graph) illustrates the group data for the effect of MS procaine on wheel-running induced by $\mathrm{PH}$ stimulation. Before the microinfusion of procaine into the MS, increasing intensities of $\mathrm{PH}$ stimulation induced significantly increasing wheel-running speeds in the animals $\left(F_{(2,30)}=14.4, p<0.0001\right)$. PH stimulation at the low intensity $(0.28 \mathrm{~mA})$ caused a wheel-running speed of 5.8 $\pm 0.7 \mathrm{~km} / \mathrm{hr}, 10.9 \pm 1.2 \mathrm{~km} / \mathrm{hr}$ at the medium intensity $(0.41 \mathrm{~mA})$, and $15.7 \pm 1.9 \mathrm{~km} / \mathrm{hr}$ at the high intensity $(0.54 \mathrm{~mA})$. After the microinfusion of procaine into the MS (P5), wheel-running was totally abolished in all animals $(11 / 11)$ at the low intensity, in 8 of 11 animals at the medium intensity, and in 7 of 11 animals at the highest intensity (bottom graph, right of dashed line). A repeatedmeasures ANOVA revealed that running speeds were significantly reduced $\left(F_{(5,150)}=41.2, p<0.0001\right)$, causing a running speed of zero at the lowest intensity $(0.28 \mathrm{~mA})$, a mean running speed of $1.6 \pm 0.9 \mathrm{~km} / \mathrm{hr}$ at the medium intensity $(0.41 \mathrm{~mA})$, and $3.6 \pm 1.6 \mathrm{~km} / \mathrm{hr}$ at the highest intensity $(0.54 \mathrm{~mA})$ of $\mathrm{PH}$ stimulation in the P5 condition. Figure 3 also shows that in the P15-P60 conditions, running speeds gradually recovered to PRE values. $\mathrm{PH}$ stimulation at the highest intensity induced running speeds of $9.4 \pm 1.8 \mathrm{~km} / \mathrm{hr}$ at $\mathrm{P} 15,12.5 \pm 1.9 \mathrm{~km} / \mathrm{hr}$ at $\mathrm{P} 30,13.2 \pm 1.9 \mathrm{~km} / \mathrm{hr}$ at $\mathrm{P} 45$, and $13.9 \pm 1.9 \mathrm{~km} / \mathrm{hr}$ at $\mathrm{P} 60$. By the $\mathrm{P} 60$ condition, running speeds induced by $\mathrm{PH}$ stimulation were no longer significantly different from the PRE condition, indicating that the MS had recovered from the procaine microinfusion $(15.7 \pm 1.9 \mathrm{~km} / \mathrm{hr}$ PRE vs $13.9 \pm 1.9 \mathrm{~km} / \mathrm{hr} \mathrm{P} 60$ at the highest stimulus level; $t_{(10)}=$ $1.4, p=0.19$ ).

Those animals in which HPC- $\theta$ was recorded during PII stimulation in the P5 condition were the same animals that showed wheel-running behavior, with one exception. Two additional animals in which no prominent theta peaks appeared in the FFT analyses did show wheel-running behavior at reduced speeds compared with PRE values.

\section{Saline and histological controls}

Three animals received injections of physiological saline $(\mathrm{NaCl})$ into the MS before the microinfusion of procaine. Table 1 presents the results of these microinfusions. The microinfusion of $\mathrm{NaCl}$ into the MS had no effect on the amplitude and peak frequency of HPC- $\theta$ or on wheel-running speeds induced during low-, medium-, or high-intensity PH stimulation. Figure 4 presents the results of two animals that received microinfusions of procaine in the lateral septal nucleus (LS) and the paraventricular thalamic nuclcus (PVT). The top illustrations reconstruct coronal sections that show the location of the procaine microinfusion cannula tips in these animals. The bottom graphs present the results of a $2.5 \mu \mathrm{l}$ infusion in the LS and $5.0 \mu \mathrm{l}$ infusion in the PVT on the amplitude and peak frequency of HPC- $\theta$ and the running speed induced at the highest level of PH stimulation. The graphs show no significant difference between the pre- and postprocaine values for these measures, with the exception of the LS microinfusion, which revealed a significant reduction in the amplitude of HPC- $\theta$ (51.2 
Table 1. The result of physiological saline microinfusion into the MS on the amplitude, frequency, and speed of wheel-running

\begin{tabular}{|c|c|c|c|c|c|}
\hline & PH stimulus intensity & Pre- $\mathrm{NaCl}$ & Post- $\mathrm{NaCl}$ & $t$-value $(\mathrm{df}=2)$ & $p$ \\
\hline \multirow[t]{3}{*}{ Amplitude of theta $(\mathrm{dB})$} & low & $40.3 \pm 6.3$ & $38.0 \pm 4.9$ & 0.73 & 0.54 \\
\hline & medium & $40.7 \pm 5.9$ & $43.3 \pm 4.3$ & -0.78 & 0.52 \\
\hline & high & $46.3 \pm 6.7$ & $41.7 \pm 5.7$ & 3.6 & 0.07 \\
\hline \multirow[t]{3}{*}{ Peak frequency of theta $(\mathrm{Hz})$} & low & $8.1 \pm 0.3$ & $8.3 \pm 0.2$ & -0.56 & 0.63 \\
\hline & medium & $8.8 \pm 0.3$ & $8.6 \pm 0.4$ & 4.0 & 0.06 \\
\hline & high & $9.1 \pm 0.3$ & $9.1 \pm 0.3$ & 0.189 & 0.87 \\
\hline \multirow[t]{3}{*}{ Wheel-running speed $(\mathrm{km} / \mathrm{hr})$} & low & $7.1 \pm 1.3$ & $8.5 \pm 4.3$ & -0.39 & 0.73 \\
\hline & medium & $16.8 \pm 1.9$ & $15.5 \pm 3.9$ & 0.54 & 0.64 \\
\hline & high & $19.4 \pm 3.3$ & $18.4 \pm 4.0$ & 0.44 & 0.70 \\
\hline
\end{tabular}

$\pm 0.2 \mathrm{~dB}$ PRE vs $43.8 \pm 1.5 \mathrm{~dB}$ postprocaine). However, after the LS procaine microinfusion, the frequency of HPC- $\theta$ and the running speed elicited by $\mathrm{PH}$ stimulation remained unchanged.

Finally, to examine the relationship between HPC- $\theta$ and wheelrunning behavior, multiple-regression analysis was used to determine whether the frequency or amplitude of HPC- $\theta$ (independent and uncorrelated variables; frequency vs amplitude of HPC- $\theta$, $F_{(1,9)}=2.2, p=0.17$ ) accounted for a significant proportion of variance in the speed of wheel-running (the dependent variable). Regression analysis revcaled that the frequency of HPC- 0 accounted for a significant proportion of variance in the speed data $(t=4.0$, $p=0.0002)$, whereas the amplitude of HPC- $\theta$ did not $(t=-0.589$, $p=0.5592$ )

\section{DISCUSSION}

A new and important finding regarding the behavioral correlates of HPC- $\theta$ has emerged from the present study. This experiment clearly demonstrates that the wheel-running behavior induced by electrical stimulation of the $\mathrm{PH}$, and the simultaneously occurring HPC- $\theta$, is caused by activation of the ascending hypothalamoseptal pathway. Thus, the motor behavior induced by PH electrical stimulation appears to depend critically on the ascending activation of neural circuitry in the septo-hippocampal structures underlying the generation of theta field activity - not on descending activation of brainstem and/or peripheral motor systems. The PH has been shown to comprise a critical part of the ascending hippocampal synchronizing system in urethane-anesthetized rats (Colom et al., 1987; Bland et al., 1994; Oddie et al., 1994). Furthermore, the hippocampal activation caused by electrical stimulation of the $\mathrm{PH}$ in urethane-anesthetized rats is dependent on the integrity of the medial septum (Smythe et al., 1991). The current findings are the first to demonstrate that the integrity of the MS is necessary for modulating both the locomotor activity and the concomitant HPC- $\theta$ induced by electrical stimulation of the $\mathrm{PH}$ in the freely moving animal.

Before the infusion of procaine into the MS (MS inactivation), PH stimulation elicited HPC- $\theta$ and running behavior in rats placed in a running-wheel. In addition, there was a positive linear relationship among the intensity of electrical stimulation of the $\mathrm{PH}$, running speeds, and peak theta frequencies, confirming the previous results of Bland and Vanderwolf (1972a). MS inactivation then abolished PH stimulation-induced wheel-running behavior and the simultaneously occurring HPC- $\theta$. Immediately after MS inactivation, the animals initially appeared catatonic (less likely to initiate motor behavior). Mizumori et al. (1989) and Lawson and Bland (1993) previously demonstrated that MS inactivation in freely moving rats abolished HPC- $\theta$ and reduced the amount of spontaneously occurring locomotor activity. The ani- mals in this experiment showed normal motor activity when handled, but electrical stimulation of the $\mathrm{PH}$ was no longer capable of inducing wheel-running behavior after procaine inactivation of the MS. However, PH stimulation did elicit hippocampal sharpwave activity with no overt behavior changes during this time period. Hippocampal sharp-wave activity and HPC- $\theta$ have been shown previously to be mutually antagonistic (Lerma et al., 1984; Suzuki and Smith, 1987). As the MS recovered from the procaine microinfusion, $\mathrm{PH}$ stimulation again elicited wheel-running behavior and HPC- $\theta$, although at significantly reduced running speeds and peak theta frequencies, respectively. The amplitude of HPC- $\theta$ was also significantly decreased but recovered to PRE values before the recovery of peak theta frequencies. Sixty minutes after the procaine infusion in MS, the amplitude, frequency, and speed of wheel-running elicited by $\mathrm{PH}$ stimulation had all returned to PRE values. In addition, a multiple-regression analysis revealed that the frequency, and not the amplitude, of HPC- $\theta$ accounted for a significant proportion of variance in the running speed data. This suggested that the frequency of HPC- $\theta$, as opposed to the amplitude, was more critically related to the speed of the elicited wheel running behavior.

Many studies reinforce the notion that brain regions involved in the production of synchronous field activity play a role in the organization of motor behavior. Lesions of the hippocampal formation consistently produce hyperactivity in animals (for review, see Gray, 1982). MS lesions have been shown to cause hyperactivity that was attributed to the perseveration of body turns (McNaughton and Feldon, 1984). Conversely, MS inactivation initially causes the reduction of voluntary motor behavior (Mizumori et al., 1989; Lawson and Bland, 1993) until animals are forced to move, after which the animals appear to move normally. Moreover, PH lesions reduce the occurrence of voluntary behaviors while leaving "automatic" behaviors intact (Robinson and Whishaw, 1974). These studies are consistent with the notion that lesions at different levels of the ascending brainstem hippocampal synchronizing pathway influence motor behavior.

Numerous studies also demonstrate that manipulations that affect hippocampal synchrony also influence locomotor activity and vice versa. For example, in animals that must jump to avoid foot-shock, the frequency and amplitude of HPC- $\theta$ increased as the height that the animal must jump increased (Whishaw and Vanderwolf, 1973; Morris and Hagan, 1983). In this and previous studies noted, PH stimulation that elicits HPC- $\theta$ also induces motor behavior. The same stimulation parameters that induce HPC- $\theta$ and motor behavior during PH stimulation arrest movement when delivered to the dentate gyrus or MS and desynchronize hippocampal field activity (Bland and Vanderwolf, 1972b; 

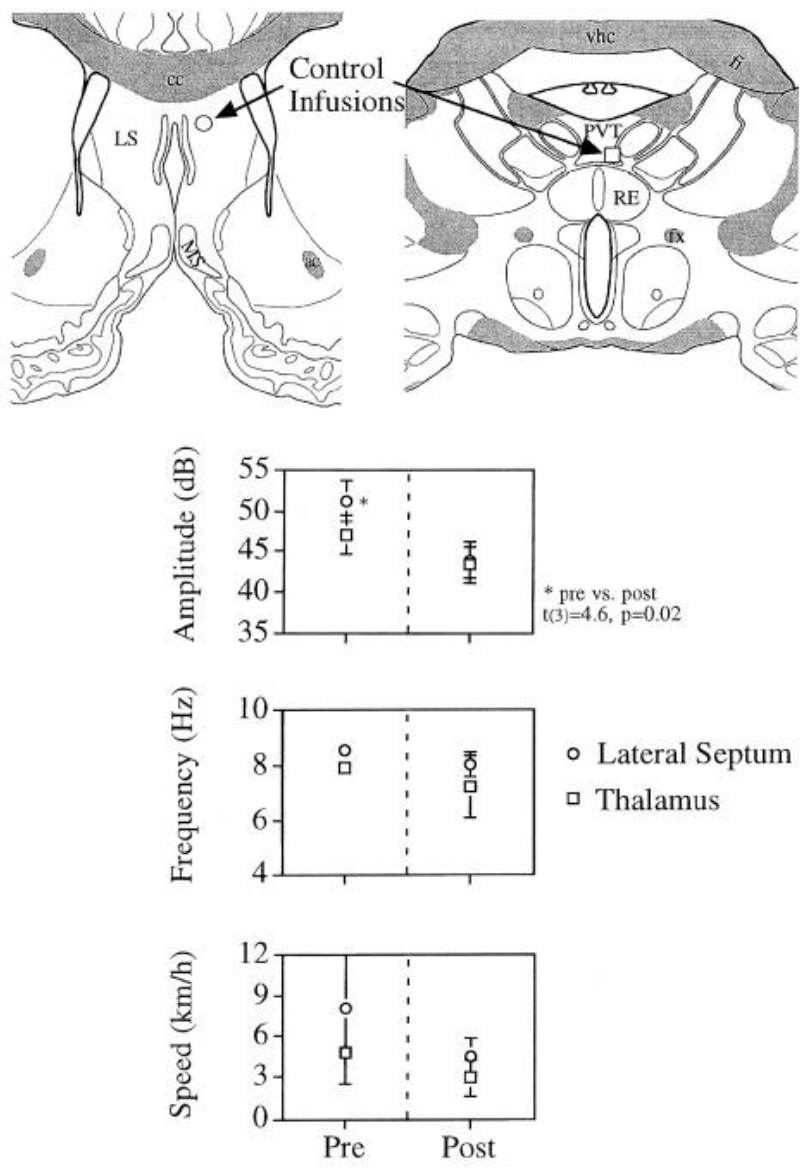

Figure 4. Procaine infusion controls. The effect of procaine microinfusion into the LS (open circle) and PVT (open square). The illustrations at the top are diagrammatic reconstructions illustrating the location of the microinfusion cannula tips in animals that did not receive procaine in the $M S$, but received procaine infusions in the lateral septum (top left) and thalamus (top right). The graphs at the bottom present the amplitude (top graph) and peak frequency (middle graph) of HPC- $\theta$ as well as the running speed elicited by the highest intensity of electrical stimulation in the $\mathrm{PH}$ before the infusion of procaine (left of dashed line, Pre) and $5 \mathrm{~min}$ after the infusion (right of dashed line, Post). The procaine infusion in either site had no effect on HPC- $\theta$, with one exception. Procaine in the lateral septum significantly decreased the amplitude of HPC- $\theta$, but did not change the peak frequency of theta or the running speed elicited by PH stimulation. $a c$, Anterior commissure; $c c$, corpus callosum; $f$, fimbria; $f x$, fornix; $L S$, lateral septal nucleus; $P V T$, paraventricular nucleus thalamus; $R E$, nucleus reunions; $v h c$, ventral hippocampal commissure.

Whishaw and Nikkel, 1975). Injections of carbachol, a cholinergic agonist, into the dentate gyrus (Mogenson and Nielson, 1984) or the MS (Monmaur and Breton, 1991) induce HPC- $\theta$ and locomotor activity. Interestingly, the locomotor activity elicited by the intrahippocampal infusion of carbachol can be blocked by injections of a glutamate antagonist into the ipsilateral nucleus accumbens (Mogenson and Nielson, 1984). Whishaw and Mittleman (1991) demonstrated that the heightened locomotor activity typical of hippocampectomized rats can be blocked by lesions of the nucleus accumbens. Finally, Lopes da Silva et al. (1985) have suggested that the hippocampus controls movement via a cornu ammonis-subiculum-nucleus accumbens-ventral pallidum-substantia nigra pars compacta pathway. Consistent with these findings is the notion that the hippocampal output may inhibit a motor inhibition system that originates in the nucleus accumbens. The current findings, taken collectively with the studies noted above, provide support for the notion that synchronous output from the hippocampal formation to motor systems plays a role in the organization of motor behavior.

Evidence has accumulated suggesting that there are two distinct forms of HPC- $\theta$ in freely moving animals. HPC- $\theta$ that occurs concomitantly with motor behavior has been referred to as type 1 $\theta$ (Vanderwolf, 1969), has been postulated to be serotonergic (Vanderwolf et al., 1985; Vanderwolf and Baker, 1986), and is abolished by general anesthetics. Several authors (Bland et al., 1984; Leung, 1984) have provided evidence that a second, cholinergically mediated type of theta (called type $2 \theta$ ) occurs coincidentally with type $1 \theta$. When animals are in a state of "alert" immobility (in a sensory processing mode), type $2 \theta$ can be recorded in isolation from type $1 \theta$ (Bland, 1986). This has been demonstrated in immobile rats preparing to initiate a jump in a jump-avoidance task (Whishaw and Vanderwolf, 1973), in immobile animals placed in close proximity to predators (Sainsbury and Montoya, 1984; Sainsbury et al., 1987), and in immobile rabbits during the processing of relevant sensory stimuli; however, as the animal habituates to a repetitive sensory stimulus, so does the prevalence of HPC- $\theta$ (Vinogradova, 1975; Whishaw and Dyck, 1984). Type $2 \theta$ is primarily cholinergic and resistant to general anesthetics (Kramis et al., 1975) (for review, see Bland, 1986). Thus, the type 1 and type $2 \theta$ systems appear to be distinct in their function and pharmacology.

What role does the neural circuitry underlying HPC- $\theta$ play in the organization of sensorimotor behavior in freely moving animals? Bland (1986) proposed that the hippocampal formation might serve to integrate sensory and motor behavior. In the proposed model, type $2 \theta$ is initiated by sensory inputs that are processed within the context of being relevant to the subsequent initiation of voluntary movements. Functionally, type $2 \theta$ provides the motor system with a "readiness" or priming signal, for both the preparation of movement per se and the intensity of its initiation. As discussed above, recent work suggests that the anatomical basis of the type $2 \theta$ system is represented in large part by the pathways ascending from $\mathrm{PO}$, through the midline posterior hypothalamus ( $\mathrm{PH}$ and supramamillary nucleus), to the medial septum and hippocampal formation. The present work, carried out in the freely moving animal, provides very clear and substantive support for this suggestion. Ongoing motor behavior would be reflected in the frequency and amplitude of type 1 HPC- $\theta$, and changes that are required in ongoing motor activity would also be signaled by the coincidentally occurring type 2 system. The inputs for type $1 \theta$ originate as feedback from motor systems to the hippocampal formation. The final result would be a change in the organization or intensity of an ongoing motor program. Thus, the hippocampal formation could serve a functional role in sensorimotor integration.

Although the testing of the sensorimotor integration model based exclusively on PH elicited HPC- $\theta$ and locomotor activity must be considered cautiously, the present study clearly indicates that the relationship between HPC- $\theta$ and voluntary motor behavior is not a spurious one. That is, the locomotor behavior elicited by $\mathrm{PH}$ stimulation is clearly dependent on the activation of ascending projections to the hippocampal formation via the medial septum. The alternative hypothesis, that $\mathrm{PH}$ induced motor behavior is attributable to the direct activation of descending projections to the brainstem and spinal motor systems, is not tenable. The findings here are consistent with the notion that locomotor activation and the simultaneously occurring HPC- $\theta$ are related and are caused by activation of the ascending brainstem pathway, 
which synchronizes hippocampal field activity and directly affects motor behavior. Future studies designed to test the hypotheses generated by sensorimotor integration theory may further clarify the relationship between HPC- $\theta$ and voluntary motor behavior.

\section{REFERENCES}

Alonso A, De Curtis M, Llinas R (1990) Postsynaptic Hebbian and non-Hebbian long-term potentiation of synaptic efficacy in the entorhinal cortex in slices and in the isolated adult guinea pig brain. Proc Natl Acad Sci USA 87:9280-9284.

Andersen P, Bland BH, Myhrer T, Schwartzkroin PA (1979) Septohippocampal pathway necessary for dentate theta production. Brain Res 165:13-22.

Bennett TL, Herbert PN, Nunn DE (1973) Hippocampal theta activity and the attention component of discriminative learning. J Behav Biol $8: 173-181$.

Black AH (1975) Hippocampal electrical activity and behavior. In: The hippocampus, Vol 2, Neurophysiology and behavior (Isaacson RL, Pribram KH, eds), New York: Plenum.

Bland BH (1986) The physiology and pharmacology of hippocampal formation theta rhythms. Prog Neurobiol 26:1-54.

Bland BH, Colom LV (1993) Extrinsic and intrinsic properties underlying oscillation and synchrony in limbic cortex. Prog Neurobiol 41:157-208.

Bland BH, Vanderwolf CH (1972a) Diencephalic and hippocampal mechanisms of motor activity in the rat: effects of posterior hypothalamic stimulation on behavior and hippocampal slow wave activity. Brain Res 43:67-88.

Bland BH, Vanderwolf CH (1972b) Electrical stimulation of the hippocampal formation: behavioural and bioelectric effects. Brain Res 43:89-106.

Bland BH, Oddie SD, Colom LV, Vertes RP (1994) Extrinsic modulation of medial septal cell discharges by the ascending brainstem hippocampal synchronizing pathway. Hippocampus 4:649-660.

Bland BH, Seto MG, Sinclair BR, Fraser SM (1984) The pharmacology of hippocampal theta cells: evidence that the sensory processing correlate is cholinergic. Brain Res 229:121-131.

Bland BH, Konopacki J, Kirk IJ, Oddie SD, Dickson CT (1995) Discharge patterns of hippocampal theta-related cells in the caudal diencephalon of the urethane-anesthetized rat. J Neurophysiol 74:322-333.

Brücke F, Petsche H, Pillat B, Deisenhammer E (1959) Uber veranderungen des hippocampus-elektrencephalogrammes beim kaninchen nach novocainjektion in die septumnucleus. Arch Exp Path Pharmakin 273:276-284.

Buzsaki G (1989) Two-stage model of memory trace formation: a role for "noisy" brain states. Neuroscience 31:551-570.

Buzsaki G, Leung LWS, Vanderwolf CH (1983) Cellular bases of hippocampal EEG in the behaving rat. Brain Res Rev 6:139-171.

Colom LV, Ford RD, Bland BH (1987) Hippocampal formation neurons code the level of activation of the cholinergic septohippocampal pathway. Brain Res 410:12-20.

Donovick PJ (1968) Effects of localized septal lesions on hippocampal EEG activity and behavior in rats. J Comp Physiol Psychol 66:569-578.

Eichenbaum H, Otto T, Cohen NJ (1992) The hippocampus: what does it do? Behav Neur Biol 57:2-36.

Elazar A, Adey WR (1967) Spectral analysis of low frequency components in the electrical activity of the hippocampus during learning. Electroencephalogr Clin Neurophysiol 23:225-240.

Gatztelu JM, Romero-Vives M, Abraira V, Garcia-Austt E (1994) Hippocampal EEG theta power density is similar during slow-wave sleep and paradoxical sleep: a long-term study in rats. Neuroscience Lett $172: 31-34$

Gerben MJ (1968) Running elicited by hypothalamic stimulation. Psychon Sci 12:19-20.

Gerben MJ (1969) Elicited locomotor behavior during hypothalamic self-stimulation. Commun Behav Biol 3:223-231.

Grastyan E, Lissak K, Madarasz I, Donhoffer H (1959) The hippocampal electrical activity during the development of conditioned reflexes. Electroencephalogr Clin Neurophysiol 11:409-430.

Grastyan E, Karmos G, Vereckey L, Kelleny IL (1966) The hippocampal electrical correlates of the homeostatic regulation of motivation. Electroencephalogr Clin Neurophysiol 21:34-53.
Gray JA (1971) Medial septal lesions, hippocampal theta rhythm and the control of vibrissal movement in the freely moving rat. Electroencephalogr Clin Neurophysiol 30:189-197.

Gray JA (1982) The neuropsychology of anxiety: an inquiry into the functions of the septo-hippocampal system. Oxford: Clarendon.

Green JD, Arduini AA (1954) Hippocampal electrical activity in arousal. J Neurophysiol 17:533-557.

Greenstein YJ, Pavlides C, Winson J (1988) Long-term potentiation in the dentate gyrus is preferentially induced at theta rhythm periodicity. Brain Res 438:331-334.

Huerta PT, Lisman JE (1993) Heightened synaptic plasticity of hippocampal CA1 neurons during a cholinergically induced rhythmic state. Nature 364:723-725.

Kirk IJ, McNaughton N (1991) Supramammillary cell firing and hippocampal rhythmical slow activity. NeuroReport 2:723-725.

Kirk IJ, McNaughton N (1993) Mapping the differential effects of procaine on the frequency and amplitude of reticularly elicited rhythmical slow activity. Hippocampus 3:517-526.

Klemm WR (1972a) Ascending and descending influences in the brainstem reticulum: a re-examination. Brain Res 36:444-452.

Klemm WR (1972b) Effects of electronic stimulation of brainstem reticular formation on hippocampal theta rhythm and muscle activity in unanesthetized, cervical and midhrain-transected rats. Brain Res 41:331-344.

Kocsis B, Vertes RP (1994) Characterization of neurons in the supramamillary nuclcus and mamillary body that discharge rhythmically with the hippocampal theta rhythm in the rat. J Neurosci 14:7040-7052.

Kolb B, Whishaw IQ (1977) Effects of brain lesions and atropine on hippocampal and neocortical electroencephalograms in the rat. Exp Neurol 56:1-22.

Kramis R, Vanderwolf CH, Bland BH (1975) Two types of hippocampal rhythmical slow activity in both the rabbit and the rat: relations to behavior and effects of atropine, diethyl ether, urethane, and pentobarbital. Exp Neurol 49:58-85.

Lawson VH, Bland BH (1993) The role of the septohippocampal pathway in the regulation of hippocampal field activity and behavior: analysis by the intraseptal microinfusion of carbachol, atropine, and procaine. Exp Neurol 120:132-144.

Lerma J, Herreras O, Monoz D, Solis JM (1984) Interactions between hippocampal penicillin spikes and theta rhythm. Electroencephalogr Clin Neurophysiol 57:532-540.

Leung LS (1984) Pharmacology of theta phase shift in the hippocampal CA1 region of freely moving rats. Electroencephalogr Clin Neurophysiol 60:457-466.

Lopes da Silva FH, Groenewegen HJ, Holsheimer J, Room P, Witter MP, Van Groen T, Wadman WJ (1985) The hippocampus as a set of partially overlapping segments with a topographically organized system of inputs and outputs: the entorhinal cortex as a sensory gate, the medial septum as a gain-setting system and the ventral striatum as a motor interface. In: Electrical activity of the archicortex (Buzsaki G, Vanderwolf $\mathrm{CH}$, eds), Budapest: Adademiai Kiado.

Macadar SW, Chalupa LM, Lindsley DB (1974) Differentiation of brainstem loci which affect hippocampal and neocortical electrical activity. Exp Neurol 120:132-144.

Markowska AL, Olton DS, Givens B (1995) Cholinergic manipulations in the medial septal area: age related effects on working memory and hippocampal electrophysiology. J Neurosci 15:2063-2073.

McNaughton N, Feldon J (1980) Spontaneous alternation of body turns and place: differential effects of amylobarbitone, scopolamine and septal lesions. Psychopharmacology 68:201-206.

Mizumori SJY, Barnes CA, McNaughton BL (1989) Reversible inactivation of the medial septum: selective effects on the spontaneous unit activity of different hippocampal cell types. Brain Res 500:99-106.

Mogenson G.J, Nielsen M (1984) A study of the contribution of hippocampal-accumbens-subpallidal projections to locomotor activity. Behav Neurol Biol 42:38-51.

Morris RGM, Hagan JJ (1983) Hippocampal clectrical activity and ballistic movement. In: Neurobiology of the hippocampus (Seifert W, ed), London: Academic.

Monmaur P, Breton P (1991) Elicitation of hippocampal theta by intraseptal carbachol injection in freely moving rats. Brain Res 544:150-155.

Monmaur PL, Haucine O, Delacour J (1979) Experimental dissociation between wakefulness and paradoxical sleep hippocampal theta. Physiol Behav 23:471-479. 
Myhrer T (1975) Locomotor avoidance and maze behavior in rats with selective disruption of hippocampal output. J Comp Physiol Psychol 89:759-777.

Nunez A, DE Andres I, Garcia-Austt E (1991) Relationships of nucleus reticularis pontis oralis neuronal discharge with sensory and carbachol evoked hippocampal theta rhythm. Exp Brain Res 87:303-308.

Oddie SD, Bland BH, Colom LV, Vertes RP (1994) The midline posterior hypothalamic region comprises a critical part of the ascending brainstem hippocampal synchronizing pathway. Hippocampus 4:454-473.

O'Keefe J, Nadel L (1978) The hippocampus as a cognitive map. Oxford: Oxford UP.

Paiva T, Lopes Da Silva FH, Mollevanger W (1976) Modulating systems of hippocampal EEG. Electroencephalogr Clin Neurophysiol 40:470-480.

Pavlides D, Greenstein YI, Goudman M, Winson .J (1988) Long-term potentiation in the dentate gyrus is induced preferentially on the positive phase of theta-rhythm. Brain Res 439:383-387.

Petsche H, Stumpf C (1960) Topographic and toposcopic study of origin and spread of the regular synchronized arousal pattern in the rabbit. Electroencephalogr Clin Neurophysiol 12:589-600.

Petsche H, Stumpf G, Gogolak G (1962) The significance of the rabbit's septum as a relay station between the midbrain and the hippocampus. I. The control of hippocampus arousal activity by the septum cells. Electroencephalogr Clin Neurophysiol 19:25-33.

Ranck JB (1973) Studies on single neurons in dorsal hippocampal formation and septum in unrestrained rats. I. Behavioral correlates and firing repertoires. Exp Neurol 41:461-531.

Rawlins JNP, Feldon J, Gray JA (1979) Septo-hippocampal connections and the hippocampal theta rhythm. Exp Brain Res 37:49-63.

Robinson TE, Vanderwolf CH (1978) Electrical stimulation of the brainstem in freely moving rats. II. Effects on hippocampal and neocortical electrical activity and relations to behavior. Exp Neurol 61:485-515.

Robinson TE, Whishaw IQ (1974) Effects of posterior hypothalamic lesions on voluntary behavior and hippocampal electroencephalograms in the rat. J Comp Physiol Psychol 86:768-786.

Rose GM, Dunwiddie TV (1986) Induction of hippocampal long-term potentiation using physiologically patterned stimulation. Neurosci Lett 69:244-248.

Sainsbury RS, Bland BH (1981) The effects of selective septal lesions on theta production in CA1 and the dentate gyrus of the hippocampus. Physiol Behav 26:1097-1101.

Sainsbury RS, Montoya CP (1984) The relationship between type 2 theta and behavior. Physiol Behav 33:621-626.

Sainsbury RS, Heynen A, Montoya CP (1987) Behavioral correlates of hippocampal type 2 theta in the rat. Physiol Behav 39:513-519.

Smythe JW, Christic BR, Colom LV, Lawson VH, Bland BH (1991) Hippocampal theta field activity and theta-on/theta-off cell discharges are controlled by an ascending hypothalamo-septal pathway. J Neurosci 11:2241-2248.

Staubli U, Xu FB (1995) Effects of 5-HT 3 receptor antagonism on hippocampal theta rhythm, memory, and LTP induction in the freely moving rat. J Neurosci 15:2445-2452.

Stewart M, Fox SE (1990) Do septal neurons pace the hippocampal theta rhythm? Trends Neurosci 13:163-168.

Suzuki SS, Smith GK (1987) Spontaneous EEG spikes in the normal hippocampus. I. Behavioral correlates, laminar profiles and bilateral synchrony. Electroencephalogr Clin Neurophysiol 67:348-359.

Swanson LW (1992) Brain maps: structure of the rat brain. Amsterdam: Elsevier.
Thompson RF, Berger TW, Berry SD (1980) Brain mechanisms of learning. In: Neural mechanisms of goal-directed behaviour and learning (Thompson RF, Hicks LH, Shvyrkov VB, cds), New York: Academic.

Torii S (1961) Two types of pattern of hippocampal electrical activity induced by stimulation of hypothalamus and surrounding parts of rabbits brain. Jpn J Physiol 11:147-157.

Vanderwolf CH (1969) Hippocampal electrical activity and voluntary movement in the rat. Electroencephalogr Clin Neurophysiol 26:407-418.

Vanderwolf CH, Leung LWS, Cooley RK (1985) Pathways through cingulate neo- and entorhinal cortices mediate atropine-resistant hippocampal rhythmical slow activity. Brain Res 347:58-73.

Vertes RP (1980) Brainstem activation of the hippocampus: a role for the magnocellular reticular formation of the mlf. Electroencephalogr Clin Neurophysiol 50:48-58.

Vertes RP (1981) An analysis of ascending brain stem systems involved in hippocampal synchronization and desynchronization. J Neurophysiol 46:1140-1159.

Vertes RP (1982) Brainstem generation of the hippocampal EEG. Prog Neurobiol 19:159-186.

Vertes RP (1984) A lectin horseradish peroxidase study of the origin of ascending fibers in the medial forebrain bundle of the rat: the lower brainstem. Neuroscience 11:651-668.

Vertes RP (1988) Brainstem afferents to the basal forebrain in the rat. Neuroscience 24:907-935.

Vertes RP (1992) PHA-L analysis of projections from the supramammillary nucleus in the rat. J Comp Neurol 326:595-622.

Vertes RP, Crane AM, Colom LV, Bland BH (1995) PHA-L analysis of ascending projections from the posterior hypothalamus in the rat. $\mathrm{J}$ Comp Neurol 359:90-116.

Vinogradova OS (1975) Functional organization of the limbic system in the process of registration of information: facts and hypotheses. In: The hippocampus, Vol 2, Neurophysiology and behavior (Isaacson RL, Pribram KH, eds), pp 1-70. New York: Plenum.

Whishaw IQ, Dyck R (1984) The comparative potency of tactile vs. auditory and visual stimulus repetition in eliciting activated forebrain EEG in the rabbit. Behav Neurosci 26:351-358.

Whishaw IQ, Mittleman G (1991) Hippocampal modulation of nucleus accumbens: behavioral evidence from amphetamine-induced activity profiles. Behav Neural Biol 43:28-35.

Whishaw IQ, Nikkel RW (1975) Anterior hypothalamic electrical stimulation and hippocampal EEG in the rat: suppressed EEG, locomotion, self-stimulation and inhibition of shock avoidance. Behav Biol 13:1-20.

Whishaw IQ, Vanderwolf CH (1973) Hippocampal EEG and behavior: changes in amplitude and frequency of RSA (thea rhythm) associated with spontaneous and learned movement patterns in rats and cats. $J$ Behav Biol 8:461-484.

Whishaw IQ, Bland $\mathrm{BH}$, Vanderwolf $\mathrm{CH}$ (1972) Relations among hippocampal activity, sclf-stimulation, cvoked bchavior, and heart rate during electrical stimulation of the lateral hypothalamus. J Comp Physiol Psychol 79:115-127.

Winson J (1978) Loss of hippocampal theta rhythm results in spatial memory deficit in the rat. Science 210:160-163.

Yokota T, Fujimori B (1964) Effects of brainstem stimulation upon hippocampal electrical activity, somatomotor reflexes and autonomic functions. Electroencephalogr Clin Neurophysiol 16:375-382. 